\title{
Disfunção sexual feminina em idade reprodutiva - prevalência e fatores associados
}

Bárbara Ribeiro, ${ }^{*}$ Ana Teresa Magalhães, ${ }^{* *}$ Ivone Mota**

\section{RESUMO}

Objetivos: A disfunção sexual feminina (DSF) é altamente prevalente, situando-se entre os $40 \%$ e $70 \%$ em Portugal, e traduz-se por uma alteração em qualquer uma das fases do ciclo de resposta sexual da mulher (desejo, excitação e orgasmo) ou ainda por perturbações dolorosas associadas ao ato sexual. O presente estudo pretendeu estudar a prevalência da DSF numa amostra de mulheres em idade reprodutiva, a prevalência dos diferentes subtipos de DSF e a existência de fatores associados.

Tipo de estudo: Tratou-se de um estudo observacional, transversal e analítico.

Local: Unidades de Saúde Familiar (USF) Novo Cuidar, Centro de Saúde de Fafe.

População: Mulheres entre os 18 e os 58 anos utentes da USF Novo Cuidar.

Métodos: A uma amostra aleatória de 346 utentes foi aplicado um questionário anónimo e confidencial de autorresposta. Usaram-se os testes Qui-Quadrado ou de Fisher para comparar proporções, o Odds Ratio para determinar a força de associação entre variáveis e os testes $t$-Student e Mann-Whitney para testar a associação entre variáveis qualitativas e quantitativas (adotou-se um nível de significância de 0,05 ).

Resultados: A taxa de resposta foi de $86,4 \%$ e a prevalência de DSF foi de $77,2 \%$ (IC95\% 72,0-82,7), tendo a perturbação do orgasmo sido o subtipo mais prevalente, 55,8\% (IC95\% 51,0-63,9). Foi encontrada uma associação entre a contraceção hormonal e a perturbação do desejo $(p=0,003)$. A aversão sexual foi estatisticamente relacionada com experiências sexuais indesejadas prévias $(p=0,001)$.

Conclusões: A DSF em idade reprodutiva é muito prevalente apesar de apenas metade das mulheres a considerarem um problema. Foram encontradas associações com alguns fatores que podem ter importância na vivência de uma sexualidade feliz pela mulher.

Palavras-chave: Disfunção Sexual; Feminina; Prevalência; Pré-menopausa; Fatores Demográficos.

\section{INTRODUÇÃO}

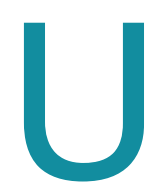

ma vida sexual satisfatória é parte integrante da saúde global do ser humano e do bem-estar individual, sendo muito importante numa relação afetiva. A sexualidade é multifatorial e influenciada por todas as dimensões do indivíduo, nomeadamente a personalidade, a biologia, $\mathrm{o}$ ciclo de vida e as experiências sexuais prévias. ${ }^{1} \mathrm{~A}$ resposta sexual feminina é complexa e foi caracterizada pela primeira vez por Masters e Jonhson, em 1966, sendo constituída por quatro fases: excitação, plateau, or-

\footnotetext{
*Interna do Ano Comum no Hospital de Braga - Escola de Ciências da Saúde, Universidade do Minho

**Internas da formação específica em Medicina Geral e Familiar - USF Novo Cuidar, Centro de Saúde de Fafe
}

gasmo e resolução. ${ }^{2}$ Em 1979, Helen Kaplan abordou a importância do desejo, como uma fase cerebral prévia e propôs um modelo trifásico: desejo, excitação e orgasmo. ${ }^{3}$ Este modelo é a base das atuais classificações da disfunção sexual feminina (DSF) que se baseiam na falência de uma ou mais fases deste ciclo. ${ }^{4,5,6}$

A disfunção sexual entende-se por toda a situação em que o indivíduo não consiga concretizar uma relação sexual ou em que esta seja insatisfatória para si e/ou para o seu companheiro. ${ }^{7}$ Esta pode caracterizar-se por uma alteração no desejo sexual, na presença ou manutenção da excitação sexual e respostas somáticas à mesma, na capacidade de obter o orgasmo, na perturbação dolorosa da função sexual ou na sobreposição de qualquer uma destas alterações. Qualquer disfunção 
sexual pode estar, ou não, associada a mal-estar, avaliado subjetivamente por cada indivíduo. ${ }^{4,5,8}$

A classificação das DSF foi amplamente discutida na comunidade científica, levando à elaboração de um painel de consenso pela Fundação Americana de Doenças Urológicas, em 1998. De acordo com a Organização Mundial de Saúde, a disfunção sexual define-se na Classificação Internacional de Doenças (CID-10) como uma incapacidade frequente, há pelo menos seis meses, de o indivíduo ter a vida sexual que desejaria e inclui várias categorias, dando ênfase aos fatores físicos que influenciam esta satisfação. ${ }^{9}$ Por outro lado, a Associação Americana de Psiquiatria, através do Manual de Diagnóstico e Estatística das Perturbações Mentais, $4^{\text {a }}$ edição (DSM-IV), define a disfunção sexual como uma desordem psicofisiológica em qualquer fase do ciclo de resposta sexual, numa vertente mais relacional e emocional, considerando ainda a disfunção sexual associada ao consumo de substâncias. ${ }^{10}$

Para uniformizar estas classificações surgiu o painel de consenso já referido, que dividiu as disfunções sexuais em quatro categorias de classificação: desejo sexual hipoactivo (DSH), onde se inclui a aversão sexual; transtornos da excitação (inclui problemas de lubrificação, relaxamento muscular, sensibilidade clitoriana, fatores psicológicos e medicação); transtornos do orgasmo e transtornos sexuais dolorosos (onde se inclui a dispareunia e o vaginismo). Este consenso definiu ainda a presença de mal-estar como fundamental ao diagnóstico de determinadas desordens. ${ }^{11}$

Existem vários fatores de risco associados a disfunção sexual, entre eles a doença neurológica, endócrina ou vascular, a cirurgia genital, o abuso sexual, o nível educacional e socioeconómico, fatores psicológicos e interpessoais (depressão, ansiedade, má relação com o parceiro), doenças físicas (cancro, infertilidade), medicação (anticoncecionais orais e antidepressivos) e alterações hormonais fisiológicas como a menopausa e a amamentação. ${ }^{1,4,5,6,6,12}$

A prevalência da disfunção sexual é elevada, ${ }^{8}$ situando-se entre os 25 e os $63 \%$ a nível mundial, mas os estudos realizados são escassos ${ }^{4} \mathrm{e}$ os dados observados são bastante heterogéneos e usam diferentes modelos de classificação. ${ }^{5,12}$ Num estudo realizado nos EUA, em 31.581 mulheres entre os 18 e os 102 anos, estimou-se que cerca de $43 \%$ apresentassem alguma queixa neste sentido. O mesmo estudo evidenciou uma prevalência de $22 \%$ de distúrbios sexuais subjetivamente associados a mal-estar. O problema sexual mais comummente identificado foi o DSH (38,7\%), tendo sido o transtorno do orgasmo o menos prevalente $(20,5 \%) .{ }^{13} \mathrm{Num}$ estudo realizado em 1.219 mulheres brasileiras obteve-se uma prevalência de $49 \%$, tendo sido o transtorno da excitação sexual o distúrbio mais comum $(26,7 \%){ }^{14}$ Existem poucos estudos na população portuguesa mas as prevalências encontradas variam de 40 a $74 \% .{ }^{5,15,16}$ Num estudo de 2005 foi estimada uma prevalência global de DSF de 56\% (35\% apresentavam DSH, 31,6\% transtornos de excitação ou orgasmo e $24,1 \%$ transtornos dolorosos na relação sexual). ${ }^{17}$ Num estudo com uma amostra de 47 mulheres, o DSH foi o problema mais comum, estimando-se uma prevalência de $40,4 \% .{ }^{15}$ Noutro estudo realizado no norte do país, numa amostra de 422 mulheres, a prevalência da disfunção sexual foi bastante mais elevada, situando-se nos $74,2 \%$, tendo sido a dispareunia o distúrbio sexual mais comum. ${ }^{5}$ A heterogeneidade destes dados revela a necessidade de se realizarem mais estudos neste âmbito e em diferentes regiões do país.

Na maioria dos estudos realizados nesta área, a prevalência da disfunção sexual aumenta com a idade..$^{8,18,19}$ No climatério e na pós-menopausa aumentam significativamente as queixas no âmbito sexual como a falta de desejo, a dor durante o coito, a falta de lubrificação e a dificuldade em atingir o orgasmo.$^{8,20}$ As mudanças hormonais associadas a esta fase do ciclo de vida, como o decréscimo de estrogénios e de testosterona (na fase pré e pós-menopausa) estão associadas a estas queixas como vários estudos demonstram..$^{8,18,20,21}$ Com a idade, diminui a frequência das relações sexuais, acompanhada contudo pela diminuição da angústia associada a este facto. ${ }^{19,21,22}$ A prevalência do DSH ronda os 40 a $50 \%$ nas mulheres pós-menopausa, ficando-se pelos 15 a $25 \%$ nas mulheres em idade reprodutiva. Foi encontrada uma prevalência de disfunção sexual de $36 \%$ num estudo que avaliou 100 mulheres entre os 20 e os 39 anos, sendo a anorgasmia a perturbação mais comum (18\%), seguida da dispareunia (13\%) e da disfunção do desejo sexual (11\%). No mesmo estudo foi encontrada associação entre o baixo nível de escolaridade e a presença de disfunção sexual, tal como noutros estudos. $^{23,24}$ 
O presente trabalho de investigação tem como objetivos determinar a prevalência global da disfunção sexual e dos seus subtipos em mulheres em idade reprodutiva e determinar se existe associação com fatores sociodemográficos, método contracetivo ou préexistência de experiencias sexuais negativas.

\section{MÉTODOS}

Tratou-se de um estudo observacional, transversal e analítico com recurso a um questionário de autorresposta, confidencial e anónimo, selecionando as participantes pelo método de amostragem aleatória a partir de uma população de 4.114 utentes do sexo feminino, entre os 18 aos 58 anos, utentes da Unidade de Saúde Familiar (USF) Novo Cuidar, do Centro de Saúde de Fafe.

Para garantir significância estatística aos resultados do estudo, a amostra foi calculada, com recurso ao programa informático Epi Info ${ }^{\mathrm{TM}}$ (disponível gratuitamente na página do Center for Disease Control and Prevention, Atlanta, USA) e utilizando a função STATCALC, tendo-se chegado a uma dimensão amostral de 346 utentes. Para este cálculo foi utilizada uma prevalência estimada de $57 \%$ (média das prevalências encontradas para a disfunção sexual em Portugal), uma precisão de $5 \%$ e um nível de confiança de $95 \%$. Através de informação constante do processo clínico ou do respetivo médico assistente foram excluídas da amostra selecionada: as utentes acamadas, internadas ou institucionalizadas, as mulheres analfabetas ou portadoras de deficiência mental e as mulheres virgens ou em menopausa. As utentes selecionadas foram convidadas a participar pessoalmente (no caso de já terem consulta agendada por outro motivo durante o período de recolha de dados) ou por envio de postal. As utentes que não aceitaram participar foram substituídas por outras também selecionadas de forma aleatória da população em estudo. Após obtenção do consentimento informado os questionários foram autopreenchidos de forma anónima e confidencial.

O questionário aplicado foi baseado na «Entrevista de Disfunções Sexuais - versão feminina» criada por Sbrocco, Weisberge Barlowem 1992 e traduzida e adaptada para a população portuguesa por Nobre et al em 2003. ${ }^{25}$ Este instrumento facilita a atribuição de diagnósticos clínicos consistentes e válidos com base nos critérios definidos pelo DSM-IV.
O questionário é constituído por 43 questões e dividido em quatro secções, levando cerca de 10 a 15 minutos a ser preenchido. A primeira parte refere-se a dados sociodemográficos (aos quais foi acrescentada pelos autores uma questão sobre contraceção), a segunda parte avalia a frequência da atividade sexual, a terceira visa a existência e caracterização de disfunção sexual e seus subtipos e a última pesquisa a existência de experiências sexuais indesejadas.

Foi considerado pelos autores: i) disfunção sexual a existência de pelo menos um subtipo de DSF; ii) disfunção sexual associada a mal-estar a existência de respostas «algum» ou «muito» na pergunta relacionada com mal-estar em cada secção; iii) perturbação do desejo sexual, as respostas «baixo» ou «muito baixo» na pergunta relativa ao interesse na atividade sexual; iv) perturbação da excitação a resposta «sim» na pergunta sobre a dificuldade em atingir a lubrificação; v) perturbação do orgasmo a resposta «sim» na pergunta relativa a dificuldade em atingir o orgasmo; vi) dispareunia a existência de dor associada à atividade sexual; vii) vaginismo a contração involuntária da parede vaginal. Foram considerados válidos os questionários com resposta às questões que avaliam a existência de cada um dos subtipos de disfunção sexual (3.1, 3.2.1, 3.3.1, 3.4.1 e 3.5.1). A escolaridade baixa foi considerada do $1^{\circ}$ ao $3^{\circ}$ ciclo e a alta desde o secundário até à pós-graduação. Em relação ao nível de atividade profissional, foram consideradas ativas as mulheres empregadas, estudantes ou domésticas e não ativas as desempregadas ou reformadas.

Para a análise dos dados obtidos, estes foram codificados e posteriormente informatizados, utilizando o programa SPSS versão 19.0. A abordagem inicial passou por análise descritiva da amostra e posteriormente foram utilizados testes estatísticos como o teste Qui-Quadrado para comparar proporções e o teste de Fisher quando uma das frequências esperadas no Qui-quadrado era menor que 5. Para determinar a força de associação entre variáveis foi utilizado o Odds Ratio (OR) com um intervalo de confiança de 95\% (nível de significância de 0,05). Foram utilizados também o teste $t$-Student e o teste de Mann-Whitney para testar a associação entre variáveis qualitativas e quantitativas com um intervalo de confiança de 95\% (nível de significância de 0,05). 


\section{RESULTADOS}

Da amostra inicial de 346 utentes foram recebidos 299 questionários, o que traduz uma taxa de resposta de $86,4 \%$. Após remoção dos questionários inválidos, obteve-se uma amostra final de 214 participantes.

A média de idades encontrada foi de 35,6 anos, sendo a idade mínima 18 anos e a máxima 57 . A maioria das mulheres era casada $(64,2 \%)$, com o $12^{\circ}$ ano de escolaridade (23,3\%) e empregada $(72,6 \%)$. A mediana do número de filhos por mulher era de 1, com o mínimo de 0 e máximo de 4 (Quadro I).

O método contracetivo mais usado pelas mulheres era o hormonal, $60,5 \%$, sendo que $11,6 \%$ das mulheres não utilizava qualquer método contracetivo. A mediana de frequência de relações sexuais mensais situavase nos 8, com um mínimo de 0 e um máximo de 30. A mediana da frequência ideal referida pelas mulheres foi de 8 relações sexuais mensais (Quadro II).

A prevalência encontrada para a DSF nesta amostra de mulheres foi de 77,2\% (IC95\% 72,0-82,7), sendo a DSF em $49,3 \%$ associada subjetivamente a mal-estar. A perturbação do orgasmo foi a mais prevalente, referida por $55,8 \%$ das mulheres (IC95\% 51,0-63,9). Dos fatores associados a esta perturbação, o stress foi o mais prevalente (30,7\%). A dispareunia foi a segunda perturbação mais referida pelas mulheres (40,9\%, IC95\% 35,6-49,0), sendo o momento referido como mais doloroso $(27,4 \%)$, «durante o ato sexual». O vaginismo registou $16,7 \%$ de prevalência (IC95\% 12,1-22,8) e as experiências sexuais indesejadas prévias, 6,5\% (IC95\% 3,4-10,6) (Quadro III).

A existência de DSF foi independente de: grau de escolaridade, profissão (Quadro IV), média de idade das mulheres, média de idade do parceiro, mediana do número de filhos por mulher e uso de contracetivo hormonal (Quadros V e VI).

A mediana da frequência mensal de relações sexuais entre as mulheres com DSF foi diferente da das mulheres sem qualquer perturbação ( $p=0,038$ ) (QuadroV).

A existência de experiências sexuais indesejadas prévias também não foi estatisticamente associada a DSF; no entanto, quase todas as mulheres com estas experiências apresentaram algum tipo de disfunção sexual (92,9\%). Pelo contrário, foi encontrada uma associação estatisticamente significativa entre a aversão sexual e a existência de uma história prévia de abuso sexual, a qual parece conferir uma probabilidade superior de vir a desen-

\begin{tabular}{|c|c|}
\hline \multicolumn{2}{|c|}{$\begin{array}{l}\text { QUADRO I. Caracterização sociodemográfica das } \\
\text { participantes. }\end{array}$} \\
\hline & n (\%) \\
\hline \multicolumn{2}{|l|}{ Estado Civil } \\
\hline Solteira & $51(23,7)$ \\
\hline Co-habitação & $12(5,6)$ \\
\hline Casada & $138(64,2)$ \\
\hline Divorciada & $10(4,7)$ \\
\hline Viúva & $4(1,9)$ \\
\hline \multicolumn{2}{|l|}{ Habilitações } \\
\hline $1^{\circ}$ ciclo & $29(13,5)$ \\
\hline $2^{\circ}$ ciclo & $36(16,7)$ \\
\hline $3^{\circ}$ ciclo & $42(19,5)$ \\
\hline Ensino secundário & $50(23,3)$ \\
\hline Universidade & $40(18,6)$ \\
\hline Pós-graduação & $16(7,4)$ \\
\hline \multicolumn{2}{|l|}{ Profissão } \\
\hline Empregada & $156(72,6)$ \\
\hline Desempregada & $31(14,4)$ \\
\hline Reformada & $4(1,9)$ \\
\hline Estudante & $15(7,0)$ \\
\hline Doméstica & $9(4,2)$ \\
\hline \multicolumn{2}{|l|}{ Número de filhos } \\
\hline 0 & $70(32,6)$ \\
\hline 1 & $63(29,3)$ \\
\hline 2 & $61(28,4)$ \\
\hline 3 & $12(5,6)$ \\
\hline 4 & $4(1,9)$ \\
\hline
\end{tabular}

volver esta disfunção ( $O R=7,818, \mathrm{IC}=95 \%)$ - (QuadroVII).

Quanto à perturbação do desejo, as mulheres que usavam contracetivo hormonal, em relação às que usavam outro método contracetivo ou nenhum, tinham uma probabilidade 2,6 vezes superior de vir a sofrer de diminuição do desejo sexual $(O R=2,598$, IC $=95 \%)$ (Quadro VIII).

\section{DISCUSSÃO}

Os objetivos propostos neste estudo foram atingidos, tendo-se obtido uma prevalência de DSF de $77,2 \%$, 


\begin{tabular}{|c|c|}
\hline \multirow{2}{*}{\multicolumn{2}{|c|}{$\begin{array}{l}\text { QUADRO II. Caracterização dos hábitos sexuais } \\
\text { do parceiro das participantes. } \\
\qquad \mid \text { n (\%) }\end{array}$}} \\
\hline & \\
\hline \multicolumn{2}{|l|}{ Método contracetivo } \\
\hline Hormonal & $130(60,5)$ \\
\hline DIU & $13(6,0)$ \\
\hline Preservativo & $21(9,8)$ \\
\hline Coito Interrompido & $7(3,3)$ \\
\hline Laqueação/vasectomia & $15(7,0)$ \\
\hline Não utilizam & $25(11,6)$ \\
\hline \multicolumn{2}{|c|}{ Frequência de relações sexuais } \\
\hline 0/mês & $15(7,7)$ \\
\hline $1-5 /$ mês & $46(21,3)$ \\
\hline $6-10 /$ mês & $71(33,1)$ \\
\hline $11-15 /$ mês & $34(15,8)$ \\
\hline $16-20 /$ mês & $25(11,6)$ \\
\hline 22 - 30/mês & $4(2,3)$ \\
\hline \multicolumn{2}{|l|}{ Idade do parceiro } \\
\hline $18-30$ & $51(23,7)$ \\
\hline $31-40$ & $63(29,4)$ \\
\hline $41-58$ & $74(34,4)$ \\
\hline
\end{tabular}

DIU: dispositivo intra-uterino

\begin{tabular}{|c|c|c|}
\hline & n (\%) & IC $95 \%$ \\
\hline DSF & $166(77,2)$ & $72,0-82,7$ \\
\hline DSF associada a «mal-estar» & $106(49,3)$ & $43,6-57,3$ \\
\hline Perturbação do desejo & $55(25,7)$ & $19,6-31,8$ \\
\hline Aversão sexual & $30(14,0)$ & $10,0-19,0$ \\
\hline Perturbação da excitação & $46(21,4)$ & $16,8-27,9$ \\
\hline Perturbação do orgasmo & $120(55,8)$ & $51,0-63,9$ \\
\hline Dispareunia & $88(40,9)$ & $35,6-49,0$ \\
\hline Vaginismo & $36(16,7)$ & $12,1-22,8$ \\
\hline Experiências sexuais indesejadas & $14(6,5)$ & $3,4-10,6$ \\
\hline
\end{tabular}

DSF: Disfunção sexual feminina

concordante com outros estudos realizados em Portugal. ${ }^{5,7}$ Quando avaliado o critério «mal-estar» associado a DSF, a prevalência encontrada diminuiu para $49,3 \%$,

\begin{tabular}{|c|c|c|c|}
\hline \multicolumn{4}{|c|}{$\begin{array}{l}\text { QUADRO IV. Prevalência de disfunção sexual feminina } \\
\text { por grau de escolaridade e atividade profissional. }\end{array}$} \\
\hline & $\mathrm{n}$ total & $\%$ com DSF & $p$ \\
\hline \multicolumn{4}{|l|}{ Escolaridade } \\
\hline Baixa & 107 & 79,4 & \multirow{2}{*}{$0,623^{*}$} \\
\hline Alta & 106 & 75,5 & \\
\hline \multicolumn{4}{|c|}{ Atividade profissional } \\
\hline Ativa & 164 & 75,6 & \multirow{2}{*}{$0,117^{*}$} \\
\hline Não ativa & 35 & 88,6 & \\
\hline
\end{tabular}

DSF: Disfunção sexual feminina

*Teste de Fisher

valor que, apesar de elevado, demonstra a desvalorização deste problema por muitas mulheres.

Os transtornos da excitação (particularmente falta de lubrificação) aumentam de $10-15 \%$ para $25-30 \%$ após a menopausa, situando-se a dispareunia nos $12-45 \%$ nestas mulheres, sendo raros na fase reprodutiva (5\%). Os problemas associados ao orgasmo parecem andar pelos $20 \%$ em todas as faixas etárias. ${ }^{20}$ No entanto, existem outros estudos que contrariam esta tendência e que consideram a DSF na idade reprodutiva como subestimada. Num estudo realizado numa população de mulheres em idade reprodutiva, a prevalência do DSH atingiu os $77 \%$, o transtorno da excitação os $62 \%$ e a dificuldade em atingir o orgasmo foi referida por $56 \%$ das mulheres..$^{13}$ Outro estudo realizado em 384 mulheres (entre os 21 e os 45 anos) estimou uma prevalência global de $68,3 \%$, verificando-se um pico etário de disfunção sexual entre os 26 e os 30 anos de idade. ${ }^{6}$

A perturbação do desejo neste estudo foi de $25,7 \%$. Contudo, não foram consideradas como diminuição do desejo sexual as respostas "médio", o que pode subestimar a real prevalência desta perturbação. A toma de anticoncecionais hormonais esteve associada a uma diminuição do desejo, o que não aconteceu com outros métodos contracetivos ou nenhum $(p=0,003)$. Esta relação já foi verificada em estudos anteriores, nomeadamente num estudo alemão com cerca de $1.000 \mathrm{mu}$ lheres. ${ }^{26}$ Alguns estudos mostram como fundamental para o desejo sexual a atração pelo parceiro e também a não existência de algumas perturbações psiquiátricas como ansiedade e depressão, ${ }^{4,13}$ tratando-se de pontos 
QUADRO V. Relação entre disfunção sexual feminina e a média de idade das mulheres e do parceiro; e entre disfunção sexual feminina e mediana do número de filhos e da frequência mensal de relações sexuais.

\begin{tabular}{l|r|r|c} 
& Com DSF & Sem DSF & P \\
\hline $\begin{array}{l}\text { Idade das mulheres } \\
\text { (média) }\end{array}$ & 35,6 & 34,6 & $0,450^{* *}$ \\
\hline $\begin{array}{l}\text { Número de filhos } \\
\text { (mediana) }\end{array}$ & 1 & 1 & $0,348^{*}$ \\
\hline $\begin{array}{l}\text { Frequência mensal de } \\
\text { relações sexuais } \\
\text { (mediana) }\end{array}$ & 8 & 12 & $0,038^{*}$ \\
\hline $\begin{array}{l}\text { Idade do parceiro } \\
\text { (média) }\end{array}$ & 37,8 & 37,5 & $0,886^{* *}$ \\
\hline
\end{tabular}

DSF: disfunção sexual feminina

*Teste de Mann-Whitney; **Teste t-Student

QUADRO VI. Relação entre disfunção sexual feminina e tipo de contraceção utilizada e entre disfunção sexual feminina e existência de história de abuso sexual.

\begin{tabular}{|c|c|c|c|}
\hline & n total & $\%$ com DSF & $p$ \\
\hline \multicolumn{4}{|c|}{ Hormonal } \\
\hline Sim & 130 & 76,9 & \multirow{2}{*}{$0,865^{*}$} \\
\hline Não & 81 & 79 & \\
\hline
\end{tabular}

\section{Experiências sexuais}

indesejadas prévias

\begin{tabular}{l|r|r|l}
\hline Sim & 14 & 92,9 & \multirow{2}{*}{$0,310^{*}$} \\
\cline { 1 - 3 } Não & 194 & 77,8 & \\
\hline
\end{tabular}

DSF: Disfunção sexual feminina

*Teste de Fisher

QUADRO VII. Relação entre existência de aversão sexual e história de abuso sexual prévio.

\begin{tabular}{c|r|c|c}
$\begin{array}{l}\text { Experiências sexuais } \\
\text { indesejadas prévias }\end{array}$ & $\mathbf{n}$ total & \% com aversão sexual & $\boldsymbol{P}$ \\
\hline Sim & 14 & 50 & 0,001* \\
\hline Não & 194 & 11,3 &
\end{tabular}

*Teste de Fisher importantes a abordar clinicamente.

A perturbação da aversão foi referida por $14 \%$ das mulheres e a da excitação por $21,4 \%$. Esta última perturbação foi avaliada somente com base em dificuldades em atingir ou manter a lubrificação, pelo que seria importante medir outras variáveis como o fluxo sanguíneo pélvico e o intumescimento da genitália externa, mais difíceis de serem autoavaliadas pela mulher. A dispareunia apresentou a segunda prevalência mais elevada (40,9\%), tendo sido mais referida a dor durante $o$ ato sexual $(27,4 \%)$. $O$ vaginismo apresentou uma prevalência de $16,7 \%$, ligeiramente inferior à de 25,5 a 34,3\%, de outros estudos. ${ }^{5,15}$ A prevalência de mulheres com experiências sexuais indesejadas no passado foi de $6,5 \%$. Estatisticamente, não foi encontrada associação entre experiências indesejadas anteriores e disfunção sexual ( $p=0,310)$, apesar de quase todas as mulheres com este background apresentarem algum subtipo de disfunção. Foi encontrada uma relação estatisticamente significativa entre aversão sexual e história de experiências sexuais indesejadas $(p=0,001)$, o que faz sentido dada a componente traumática destes acontecimentos. Alguns estudos anteriores já tinham relacionado a DSF com história de abuso sexual no passado. ${ }^{4,12} \mathrm{O}$ nível de escolaridade das mulheres e a sua posição socioeconómica já foi discutida na literatura, tendo vários estudos correlacionado positivamente o baixo nível de escolaridade com a disfunção sexual. ${ }^{1,12,13,23,24}$ No entanto, neste estudo não foi verificada associação entre o nível educacional das mulheres e uma maior prevalência de DSF, resultado similar a um estudo brasileiro de 2010. ${ }^{27}$ Neste estudo também não foi encontrada associação estatisticamente significativa entre a média de idade das mulheres, média de idade do parceiro, a mediana do número de filhos e a DSF. No entanto, seria importante no futuro indagar sobre o papel do parceiro e do relacionamento sexual e emocional no desenvolvimento de disfunções sexuais, apontado em alguns estudos como fundamental. ${ }^{4}$ A mediana de frequência mensal de relações sexuais entre as mulheres com DSF foi superior à das mulheres sem DSF, o que pode sugerir que mais importante que relações sexuais 


\begin{tabular}{|c|c|c|c|}
\hline \multicolumn{4}{|c|}{$\begin{array}{l}\text { QUADRO VIII. Prevalência da perturbação do desejo por tipo de } \\
\text { contraceção. }\end{array}$} \\
\hline Hormonal & n total & \% com perturbação do desejo & $\mathbf{P}$ \\
\hline Sim & 130 & 81,5 & \multirow{2}{*}{$0,003^{*}$} \\
\hline Não & 81 & 63 & \\
\hline
\end{tabular}

*Teste de Fisher

frequentes será o prazer tirado nas mesmas e a qualidade do relacionamento com o parceiro.

Este estudo é dos primeiros a avaliar a DSF em mulheres portuguesas somente na idade reprodutiva, o que poderia levar-nos a esperar uma prevalência menor, uma vez que a menopausa e as alterações hormonais esperadas nessa fase são comprovadamente fatores importantes para o desenvolvimento de disfunções sexuais..$^{20,21,22}$ No entanto, as prevalências encontradas foram elevadas, subvalorizando fatores hormonais e emocionais próprios da idade não reprodutiva da mulher, o que já tinha sido demonstrado em estudos realizados noutros países, onde se apuraram prevalências de DSF, antes da menopausa, na ordem dos 45 a 63\%.6.18 Alguns fatores a serem abordados em estudos futuros são a toma de alguns medicamentos (não especificados no questionário), a instabilidade da relação ou problemas sexuais entre parceiros e, uma vez que estamos a estudar mulheres em idade reprodutiva, seria interessante questionar a fase do ciclo reprodutivo em que a mulher se encontra, a importância da gravidez e a existência de filhos como fatores possivelmente associados a DSF.

A existência de múltiplos critérios de diagnóstico, ao invés da universalidade na avaliação de DSF, pode levar a um viés de classificação. Relativamente à definição de DSF e seus subtipos utilizou-se a descrita no DSM-IV, uma vez que o questionário se baseia nesta classificação. Contudo, apurou-se também o critério subjetivo «mal-estar», fundamental no diagnóstico proposto pelo consenso de 1999 da Associação Americana de Doenças Urológicas. Outra limitação do estudo prende-se com a delicadeza e intimidade do tema em questão, que pode ter levado algumas mulheres a responderem de acordo com o que pensariam ser mais aceite socialmente e não de acordo com a sua situação. No entanto, o fato de o questionário ser anónimo e de autopreenchimento tentou contornar o possível enviesamento de respostas. Outra limitação possível do estudo é a existência de um viés de seleção, ao excluírem-se as mulheres analfabetas, acamadas e institucionalizadas. Por outro lado, e apesar de a amostra ter sido selecionada aleatoriamente, o estudo dependeu da colaboração voluntária das mulheres, o que pode levar a um viés de seleção, podendo as que tiveram mais disponibilidade para se deslocarem à USF serem as que se sentiram mais confortáveis com o tema. Reconhece-se, ainda, como limitações a não formulação de hipóteses a testar a priori, assim como a não realização de uma análise multivariável (regressão logística) para determinar a força de associação entre variáveis e detetar fatores de confundimento.

Os dados obtidos neste estudo permitem-nos concluir que a DSF, tal como definida, tem uma prevalência muito elevada também na idade reprodutiva. A prevalência dos subtipos de DSF é elevada, tendo sido a disfunção do orgasmo a mais prevalente, o que nos leva a questionar o papel do parceiro e da medicação em estudos futuros. Apesar da sua difícil avaliação e da disparidade de definições de DSF, este é um problema real e que muito pode afetar a qualidade de vida das mulheres e dos seus parceiros. No entanto, um dos resultados importantes deste estudo foi que quase metade das mulheres com algum tipo de disfunção sexual não a considerava um problema, pelo que o médico deve estar atento a este problema mas também ter a perceção de que a sexualidade da mulher é complexa e multifatorial e que, se não é vista como um problema pela própria mulher, não deve ser medicalizada e colocada no espectro de doença. A DSF é actualmente um tópico controverso pela excessiva publicitação e medicalização de que tem vindo a ser alvo, particularmente pela parte da indústria farmacêutica. ${ }^{28,29}$ Nos últimos anos esta indústria tem mostrado um interesse agressivo na DSF, registando-se um crescendo de estudos, muitos deles patrocinados por essa mesma indústria. Alguns desses estudos levaram a conclusões redutoras de que este seria um problema muito prevalente e passível de tratamento farmacológico, não tendo em conta a história individual de cada mulher, a sua esfera relacional, cultural, social e psicológica. É por isso importante que o médico de Medicina Geral e Familiar tenha esta visão 
global da mulher, antes de fazer um diagnóstico ou prescrever um fármaco para este problema que, muitas vezes, necessita de uma abordagem holística e multidisciplinar.

\section{REFERÊNCIAS BIBLIOGRÁFICAS}

1. Najafabady MT, Salmani Z, Abedi P. Prevalence and related factors for anorgasmia among reproductive aged women in Hesarak, Iran. Clinics (Sao Paulo) 2011; 66 (1): 83-6.

2. Masters W, Johnson V. Human Sexual Response. Boston, MA: Little Brown; 1966.

3. Kaplan HS. The New Sex Therapy. London: Bailliere Tindall; 1974.

4. Pablo C, Soares C. As disfunções sexuais femininas. Rev Port Clin Geral 2004 Mai-Jun; 20 (3): 357-70.

5. Cerejo AC. Disfunção sexual feminina: prevalência e fatores relacionados. Rev Port Clin Geral 2006 Nov-Dez; 22 (6): 701-20.

6. Fajewonymi BA, Orji EO, Adeyemo AO. Sexual dysfunction among female patients of reproductive age in a hospital setting in Nigeria. J Health Popul Nutr 2007 Mar; 25 (1): 101-6.

7. Simões JA. Incidência de queixas de disfunção sexual na população da Rede "Médicos-Sentinela" no ano de 1998. Acta Androl 2000; 17 (2): 57-61.

8. Berman JR, Berman L, Goldstein I. Female sexual dysfunction: incidence, pathophysiology, evaluation and treatment options. Urology 1999 Sep; 54 (3): 385-91.

9. World Health Organization. The ICD-10 Classification of Mental Behavioural Disorders: Clinical Descriptions and Diagnostic Guidelines. Geneva:WHO; 2000. p. 150-152

10. American Psychiatric Association. DSM-IV. Manual de Diagnóstico e Estatística das Perturbações Mentais. $4^{\mathrm{a}}$ ed. Lisboa: Climepsi Editores; 1996. p. 505-24.

11. Basson R, Berman J, Burnett A, Derogatis L, Ferguson D, Fourcroy J, et al. Report of the Internacional Consensus Development Conference on Female Sexual Dysfunction: definitions and classifications. J Urol 2000 Mar; 163 (3): 888-93.

12. Lightner DJ. Female sexual dysfunction. Mayo Clin Proc 2002 Jul; 77 (7): 698-702.

13. Shifren JL, Monz BU, Russo PA, Segreti A, Johannes CB. Sexual problems and distress in United States women: prevalence and correlates. Obstet Gynecol 2008 Nov; 112 (5): 970-8.

14. Abdo CH, Oliveira WM Jr, Moreira ED Jr, Fittipaldi JA. Prevalence of sexual dysfunctions and correlated conditions in a sample of Brazilian women: results of the Brazilian study on sexual behavior (BSSB). Int J Impot Res 2004 Apr; 16 (2): 160-6.

15. Nobre PJ, Pinto-Gouveia J, Gomes FA. Prevalence and comorbidity of sexual dysfunctions in a Portuguese clinical sample. J Sex Marital Ther 2006 Mar-Apr; 32 (2): 173-82.

16. Gomes FA, Fonseca L, Gomes AA. Comorbilidade psiquiátrica nas disfunções sexuais, Acta Port Sexol 1997; 2 (1): 17-20.

17. Vendeira P, Pereira NM, Santo MC, Macedo A. Prevalência da disfunção sexual feminina em Portugal. Lisboa: Sociedade Portuguesa de Andrologia; 2005..

18. Lara LA, Silva AC, Romão AP, Junqueira FR. Abordagem das disfunções sexuais femininas. Rev Bras Ginecol Obstetr 2008 Jun; 30 (6): 312-21. 19. Graziottin A. Prevalence and evaluation of sexual health problems: HSDD in Europe. J Sex Med 2007 Mar; 4 (3): 211-9.

20. Gregersen N, Jensen PT, Giraldi AE. Sexual dysfunction in the peri- and postmenopause: status of incidence, pharmacological treatment and possible risks - a secondary publication. Dan Med Bull 2006 Aug; 53 (3): 349-53.

21. Dennerstein L, Dudley E, Burger $H$. Are changes in sexual functioning during midlife due to aging or menopause? Fertil Steril 2001 Sep; 76 (3): 456-60.

22. Dennerstein L, Hayes R. Confronting the challenges: epidemiological study of sexual dysfunction and menopause. J Sex Med 2005 Sep; 2 (3): 118-32.

23. Ferreira AL, Souza Al, Amorim MM. Prevalência das disfunções sexuais femininas em clínica de planejamento familiar de um hospital escola no Recife, Pernambuco. Rev Bras Saúde Materna Infantil 2007 Abr-Jun; 7 (2): 143-50.

24. Singh JC, Tharyan P, Kekre NS, Singh G, Gopalakrishnan G. Prevalence and risk factors for female sexual dysfunction in women attending a medical clinic in south India. J Postgrad Med 2009 Apr-Jun; 55 (2): 11320.

25. Nobre P. Disfunções Sexuais: Teoria, Investigação e Tratamento. Lisboa: Climepsi Editores; 2006.

26. Wallwiener CW, Wallwiener LM, Seeger H, MückAO, Bitzer J, Wallwiener $M$. Prevalence of sexual dysfunction and impact of contraception in female German medical students. J Sex Med 2010 Jun; 7 (6): 2139-48.

27. Prado D, Mota V, Lima T. Prevalência de disfunção sexual em dois grupos de mulheres de diferentes níveis socioeconómicos. Rev Brasileira Ginecol Obstetr 2010 Mar; 32 (3): 139-43.

28. Moynihan R. Merging of marketing and medical science: female sexual dysfunction. BMJ 2010 Sep 30; 341: c5050.

29. Tiefer L. Female sexual dysfunction: a case study of disease mongering and activist resistance. PLoS Med 2006 Apr; 3(4): e178.

\section{FINANCIAMENTO E CONFLITOS DE INTERESSE}

No presente estudo de investigação não são identificados conflitos de interesse de qualquer natureza.

Todas as despesas de deslocação ao centro de saúde e com os questionários utilizados para o estudo ficaram a cargo das investigadoras.

\section{ENDEREÇO PARA CORRESPONDÊNCIA}

Bárbara Cristina Carvalho Ribeiro

R. de S. José, $n^{\circ} 128,4^{\circ}$ Dto Frt,

4710-436 Braga, Portugal

E-mail: b.2xc.ribeiro@gmail.com

Recebido em 24/04/2012

Aceite para publicação em 20/02/2013 


\section{ABSTRACT}

\section{FEMALE SEXUAL DYSFUNCTION IN THE REPRODUCTIVE YEARS: PREVALENCE AND ASSOCIATED FACTORS}

Objectives: Sexual dysfunction is believed to exist in $40 \%$ to $70 \%$ of the population in Portugal. It is defined by pain or alteration of function in the phases of the sexual act. The present study was designed to estimate the prevalence of sexual dysfunction in women of reproductive age, the prevalence of the various subtypes of sexual dysfunction, and the factors associated with female sexual dysfunction.

Study design: Observational, cross-sectional and analytic study.

Setting: Novo Cuidar Family Health Unit, Fafe.

Participants: Women aged between 18 and 58 years attending the Novo Cuidar Health Unit.

Methods: An anonymous, self-administered questionnaire was given to a random sample of 346 women. The chi-squared and Fisher tests were used to compare proportions, odds ratio were computed to determine the strength of associations, and t-Student and Mann-Whitney tests were used to compare means and proportions of relevant variables.

Results: The response rate was $\mathbf{8 6 . 4 \%}$. The prevalence of female sexual dysfunction was $77,2 \%$ (C/95\% 72,0-82,7). Orgasmic disorder was the most prevalent subtype in 55,8\% (C/95\% 51,0-63,9). An association between hormonal contraception and low sexual desire was found $(p=0,003)$. Sexual aversion was statistically related with previous unwanted sexual experiences $(p=0,001)$.

Conclusions: Female sexual dysfunction in women of reproductive age is highly prevalent, although only half of the women surveyed considered it to be a problem. Significant associations were found with factors that can influence satisfying sexuality.

Key-words: Female; Sexual Dysfunction; Prevalence; Premenopause; Demographic Factors. 